\title{
BRAUER GROUP OF MODULI OF HIGGS BUNDLES AND CONNECTIONS
}

\author{
DAVID BARAGLIA, INDRANIL BISWAS, AND LAURA P. SCHAPOSNIK
}

\begin{abstract}
Given a compact Riemann surface $X$ and a semisimple affine algebraic group $G$ defined over $\mathbb{C}$, there are moduli spaces of Higgs bundles and of connections associated to $(X, G)$. We compute the Brauer group of the smooth locus of these varieties.
\end{abstract}

\section{INTRODUCTION}

We dedicate this paper to the study of the Brauer group of the moduli spaces of certain Higgs bundles and of holomorphic connections on a Riemann surface. Recall that given a complex quasiprojective variety $Z$, its Brauer group $\operatorname{Br}(Z)$ is the Morita equivalence classes of Azumaya algebras over $Z$. This group coincides with the equivalence classes of principal PGL-bundles over $Z$, where two principal PGL-bundles $P$ and $Q$ are identified if there are vector bundles $V$ and $W$ over $Z$ such that the two principal PGL-bundles $P \otimes \mathbb{P}(V)$ and $Q \otimes \mathbb{P}(W)$ are isomorphic. The cohomological Brauer group $\operatorname{Br}^{\prime}(Z)$ of the variety $Z$ is the torsion part of the étale cohomology group $H^{2}\left(Z, \mathbb{G}_{m}\right)$. There is a natural injective homomorphism $\operatorname{Br}(Z) \longrightarrow \operatorname{Br}^{\prime}(Z)$ which is in fact an isomorphism by a theorem of Gabber [dJ], [Ho].

Consider now a compact connected Riemann surface $X$ of genus $g \geq 3$. Given a fixed base point $x_{0}$ and two integers $r \geq 2$ and $\delta$, we let $\mathcal{M}_{C}$ denote the moduli space of all logarithmic connections $(E, D)$ on $X$, singular over $x_{0}$, satisfying the following four natural conditions:

I. $E$ is a holomorphic vector bundle on $X$ of rank $r$ with $\bigwedge^{r} E=\mathcal{O}_{X}\left(\delta x_{0}\right)$,

II. the logarithmic connection on $\bigwedge^{r} E=\mathcal{O}_{X}\left(\delta x_{0}\right)$ induced by $D$ coincides with the connection on $\mathcal{O}_{X}\left(\delta x_{0}\right)$ defined by the de Rham differential,

III. the residue of $D$ at $x_{0}$ is $-\frac{\delta}{r} \operatorname{Id}_{E_{x_{0}}}$, and

IV. there is no holomorphic subbundle $F \subset E$ with $1 \leq \operatorname{rank}(F)<r$ such that $D$ preserves $F$.

This moduli space $\mathcal{M}_{C}$ has a natural projective bundle once we fix a point of $X$,

$$
\mathbb{P}_{C} \longrightarrow \mathcal{M}_{C},
$$

through which in Section 2 we study the Brauer group $\operatorname{Br}\left(\mathcal{M}_{C}\right)$ :

2000 Mathematics Subject Classification. 14F22, 14H60.

Key words and phrases. Brauer group. Higgs bundles, connections, character variety. 
Theorem 2.4. The Brauer group $\operatorname{Br}\left(\mathcal{M}_{C}\right)$ is isomorphic to $\mathbb{Z} / \tau \mathbb{Z}$, where $\tau=$ g.c.d. $(r, \delta)$. The group $\operatorname{Br}\left(\mathcal{M}_{C}\right)$ is generated by the class of $\mathbb{P}_{C}$.

Fixing the compact connected Riemann surface $X$ and the invariant $\delta$, one can also compute the analytic Brauer group of the $\mathrm{SL}(r, \mathbb{C})$-character variety $\mathcal{R}$ associated to the pair $(X, \delta)$.

Theorem 2.5. The analytic cohomological Brauer group $\mathrm{Br}_{\mathrm{an}}^{\prime}(\mathcal{R})$ is isomorphic to a quotient of the cyclic group $\mathbb{Z} / \tau \mathbb{Z}$, where $\tau=$ g.c.d. $(r, \delta)$. The group $\operatorname{Br}_{\text {an }}(\mathcal{R})$ is generated by the class of a naturally associated projective bundle $\mathbb{P}_{R}$.

Over the compact connected Riemann surface $X$ one may also consider the moduli space $\mathcal{M}_{H}$ of stable Higgs bundles on $X$ of the form $(E, \Phi)$, where

- $E$ is a holomorphic vector bundle of rank $r$ with $\bigwedge^{r} E=\mathcal{O}_{X}\left(\delta x_{0}\right)$, and

- $\Phi$ is a Higgs field on $X$ with trace $(\Phi)=0$.

The moduli space $\mathcal{M}_{H}$ is a smooth quasiprojective variety which also has a natural projective bundle

$$
\mathbb{P}_{H} \longrightarrow \mathcal{M}_{H}
$$

once we fix a point of $X$. In Section 2.3 we study the Brauer group of $\mathcal{M}_{H}$ and prove the following:

Proposition 2.7. The group $\operatorname{Br}\left(\mathcal{M}_{H}\right)$ is isomorphic to the cyclic group $\mathbb{Z} / \tau \mathbb{Z}$, and it is generated by the class of $\mathbb{P}_{H}$.

One should note that, as seen in Section 3, the results of Section 2 extend to the context of principal bundles. We shall conclude this paper by looking at our results in the context of Langlands duality in Section 4.

\section{Brauer group of Some moduli spaCes}

As in the introduction, we shall consider a compact connected Riemann surface $X$ of genus $g \geq 3$ with a fixed base point $x_{0} \in X$, and denote by $K_{X}$ its canonical bundle.

2.1. Brauer group of moduli spaces of connections. A logarithmic connection on $X$ singular over $x_{0}$ is a pair of the form $(E, D)$, where $E$ is a holomorphic vector bundle on $X$ and

$$
D: E \longrightarrow E \otimes K_{X} \otimes \mathcal{O}_{X}\left(x_{0}\right)
$$

is a holomorphic differential operator of order one satisfying the Leibniz identity

$$
D(f s)=f \cdot D(s)+s \otimes(d f),
$$

for all locally defined holomorphic functions $f$ on $X$ and all locally defined holomorphic sections $s$ of $E$. Note that the fiber $\left(K_{X} \otimes \mathcal{O}_{X}\left(x_{0}\right)\right)_{x_{0}}$ is canonically identified with $\mathbb{C}$ by sending any $c \in \mathbb{C}$ to the evaluation at $x_{0}$ of the locally defined section $c \frac{d z}{z}$ of $K_{X} \otimes \mathcal{O}_{X}\left(x_{0}\right)$, where $z$ is any holomorphic function defined around $x_{0}$ with $z\left(x_{0}\right)=0$ and $(d z)\left(x_{0}\right) \neq 0$. Moreover, the evaluation of $\frac{d z}{z}$ at $x_{0}$ does not depend on the choice of the function $z$. Using this identification of $\left(K_{X} \otimes \mathcal{O}_{X}\left(x_{0}\right)\right)_{x_{0}}$ with $\mathbb{C}$, for any logarithmic 
connection $D$ as above we have the linear endomorphism of the fiber $E_{x_{0}}$ given by the composition

$$
E \stackrel{D}{\longrightarrow} E \otimes K_{X} \otimes \mathcal{O}_{X}\left(x_{0}\right) \longrightarrow\left(E \otimes K_{X} \otimes \mathcal{O}_{X}\left(x_{0}\right)\right)_{x_{0}}=E_{x_{0}} .
$$

This element of $\operatorname{End}\left(E_{x_{0}}\right)=E_{x_{0}} \otimes E_{x_{0}}^{*}$ is called the residue of $D$ (see [De, p. 53]), which we shall denote by $\operatorname{Res}\left(D, x_{0}\right)$. Then, from [Oh, pp. 16-17, Theorem 3], and [De] one has

$$
\operatorname{degree}(E)+\operatorname{trace}\left(\operatorname{Res}\left(D, x_{0}\right)\right)=0 .
$$

For notational convenience, we shall let $\mathbb{K}:=K_{X} \otimes \mathcal{O}_{X}\left(x_{0}\right)$.

Definition 2.1. A logarithmic connection $(E, D)$ as above is called semistable (respectively, stable) if for every holomorphic subbundle $0 \neq F \subsetneq E$ with $D(F) \subset F \otimes \mathbb{K}$, the following inequality holds

$$
\frac{\text { degree }(F)}{\operatorname{rank}(F)} \leq \frac{\operatorname{degree}(E)}{\operatorname{rank}(E)} \quad\left(\text { respectively }, \frac{\operatorname{degree}(F)}{\operatorname{rank}(F)}<\frac{\operatorname{degree}(E)}{\operatorname{rank}(E)}\right)
$$

As done in Section 1, fix two integers $r \geq 2$ and $\delta$, and if $g=3$, then assume that $r>2$. The holomorphic line bundle $\mathcal{O}_{X}\left(\delta x_{0}\right)$ on $X$ is equipped with the logarithmic connection given by the de Rham differential $d$. This logarithmic connection on $\mathcal{O}_{X}\left(\delta x_{0}\right)$ will be denoted by $D_{0}$. From (2.2) it follows that the residue of $D_{0}$ is $-\delta$.

In views of the notation introduced in Section 1 , let $\overline{\mathcal{M}}_{C}$ denote the moduli space of logarithmic connections $(E, D)$ on $X$, singular over $x_{0}$, satisfying the following three conditions:

I. $E$ is a holomorphic vector bundle on $X$ of rank $r$ with $\bigwedge^{r} E=\mathcal{O}_{X}\left(\delta x_{0}\right)$,

II. the logarithmic connection on $\bigwedge^{r} E=\mathcal{O}_{X}\left(\delta x_{0}\right)$ induced by $D$ coincides with $D_{0}$ defined above, and

III. $\operatorname{Res}\left(D, x_{0}\right)=-\frac{\delta}{r} \operatorname{Id}_{E_{x_{0}}}$.

Note that from $(2.2)$, the above condition on $\operatorname{Res}\left(D, x_{0}\right)$ implies that $(E, D)$ is automatically semistable. Moreover, if $\delta$ is coprime to $r$, then $(E, D)$ is in fact stable. Since the residue $\operatorname{Res}\left(D, x_{0}\right)$ is a constant multiple of $\operatorname{Id}_{E_{x_{0}}}$, the logarithmic connection on the projective bundle $\mathbb{P}(E)$ induced by $D$ is actually regular at $x_{0}$.

The above defined moduli space $\overline{\mathcal{M}}_{C}$ is a quasiprojective irreducible normal variety, defined over $\mathbb{C}$, of dimension $2\left(r^{2}-1\right)(g-1)$. Let

$$
\mathcal{M}_{C} \subset \overline{\mathcal{M}}_{C}
$$

be the Zariski open subset parametrizing the stable logarithmic connections. We note that $\mathcal{M}_{C}$ is contained in the smooth locus of $\overline{\mathcal{M}}_{C}$ (in fact, $\mathcal{M}_{C}$ is the smooth locus of the space $\left.\overline{\mathcal{M}}_{C}\right)$. We shall denote by $\operatorname{Br}\left(\mathcal{M}_{C}\right)$ the Brauer group of the smooth variety $\mathcal{M}_{C}$ which, as mentioned in Section 1 is the Morita equivalence classes of Azumaya algebras over $\mathcal{M}_{C}$. The reader should refer to [Gr1], [Gr2], [Gr3] [Mi] for the definition as well as some properties of the Brauer group.

For any $(E, D) \in \mathcal{M}_{C}$, consider any $T \in H^{0}(X, \operatorname{End}(E))$ which is flat with respect to the connection on $\operatorname{End}(E)$ induced by $D$, or equivalently, such that $D \circ T=\left(T \otimes \mathrm{Id}_{\mathbb{K}}\right) \circ D$. 
Then, for any $c \in \mathbb{C}$, the kernel of $T-c \cdot \operatorname{Id}_{E} \in H^{0}(X, \operatorname{End}(E))$ is preserved by $D$. Since $\operatorname{kernel}\left(T-c \cdot \operatorname{Id}_{E}\right)$ is either $E$ or 0 , it follows that either $T=c \cdot \operatorname{Id}_{E}$ or the endomorphism $T-c \cdot \operatorname{Id}_{E}$ is invertible. Now taking $c$ to be an eigenvalue of $T\left(x_{0}\right)$ it follows that $T=c \cdot \operatorname{Id}_{E}$. Consequently, there is a universal projective bundle

$$
\widetilde{\mathbb{P}} \longrightarrow X \times \mathcal{M}_{C}
$$

of relative dimension $r-1$ which is equipped with a relative holomorphic connection in the direction of $X$.

Definition 2.2. Given a point $x \in X$, let

$$
\mathbb{P}:=\left.\widetilde{\mathbb{P}}\right|_{\{x\} \times \mathcal{M}_{C}} \longrightarrow \mathcal{M}_{C}
$$

be the projective bundle obtained by restricting $\widetilde{\mathbb{P}}$, and denote its class by

$$
\beta \in \operatorname{Br}\left(\mathcal{M}_{C}\right) .
$$

In order to study the Brauer group $\operatorname{Br}\left(\mathcal{M}_{C}\right)$ we shall first prove the following.

Lemma 2.3. Let $Y$ be a simply connected smooth quasiprojective variety defined over the complex numbers, $W$ an algebraic vector bundle on $Y$ and

$$
q: \mathcal{W} \longrightarrow Y
$$

a torsor on $Y$ for $W$. Then the pullback homomorphism

$$
q^{*}: \operatorname{Br}(Y) \longrightarrow \operatorname{Br}(\mathcal{W})
$$

is an isomorphism.

Proof. Let $c \in H^{1}(Y, W)$ be the class of $\mathcal{W}$. Consider the extension of $\mathcal{O}_{Y}$ by $W$

$$
0 \longrightarrow W \longrightarrow \widehat{W} \stackrel{\xi}{\longrightarrow} \mathcal{O}_{Y} \longrightarrow 0
$$

associated to the cohomology class $c$. We shall denote by $1_{Y}$ the image of the section of $\mathcal{O}_{Y}$ defined by the constant function 1 on $Y$. Then, the inverse image $\xi^{-1}\left(1_{Y}\right) \subset \widehat{W}$ under the projection $\xi$ in (2.7), is a torsor on $Y$ for the vector bundle $W$. This $W$-torsor is isomorphic to the $W$-torsor $\mathcal{W}$.

Let

$$
\widehat{\eta}: P(\widehat{W}) \longrightarrow Y \text { and } \eta: P(W) \longrightarrow Y
$$

be the projective bundles on $Y$ parametrizing the lines in the fibers of $\widehat{W}$ and $W$ respectively. Note that $P(W) \subset P(\widehat{W})$, and

$$
\mathcal{W}=\xi^{-1}\left(1_{Y}\right)=P(\widehat{W}) \backslash P(W),
$$

by sending any element of $\xi^{-1}\left(1_{Y}\right)$ to the line in $\widehat{W}$ generated by it. Now from [Fo, p. 365, Lemma 0.1] and [Fo, p. 367, Theorem 1.1] we conclude that the there is an exact sequence

$$
0 \longrightarrow \operatorname{Br}(P(\widehat{W})) \longrightarrow \operatorname{Br}(\mathcal{W}) \longrightarrow H^{1}(P(W), \mathbb{Q} / \mathbb{Z}) \longrightarrow \ldots
$$


Consider the long exact sequence of homotopy groups for the fiber bundle $\eta$. The fibers of $P(W)$ are projective spaces and hence are simply connected. Since $Y$ is also simply connected, from the homotopy exact sequence for $\eta$ it follows that $P(W)$ is simply connected as well. Hence $H_{1}(P(W), \mathbb{Z})=0$, which implies that $H^{1}(P(W), \mathbb{Q} / \mathbb{Z})=0$ (universal coefficient theorem for cohomology; see [Ha, p. 195, Theorem 3.2]). Consequently, using (2.8) we conclude that

$$
\operatorname{Br}(P(\widehat{W}))=\operatorname{Br}(\mathcal{W})
$$

with the isomorphism being induced by the inclusion of $\mathcal{W}$ in $P(\widehat{W})$.

The homomorphism $\widehat{\eta}^{*}: \operatorname{Br}(Y) \longrightarrow \operatorname{Br}((P(\widehat{W}))$ induced by $\widehat{\eta}$ is an isomorphism [Ga, p. 193, Theorem 2], and the lemma follows from (2.9).

Theorem 2.4. The Brauer group $\operatorname{Br}\left(\mathcal{M}_{C}\right)$ is isomorphic to the cyclic group $\mathbb{Z} / \tau \mathbb{Z}$, where $\tau=$ g.c.d. $(r, \delta)$, and it is generated by the element $\beta$ in (2.6).

Proof. Let $\mathcal{N}$ denote the moduli space of stable vector bundles $V$ on $X$ of rank $r$ such that $\bigwedge^{r} V=\mathcal{O}_{X}\left(\delta x_{0}\right)$, which is a smooth quasiprojective irreducible complex variety of dimension $\left(r^{2}-1\right)(g-1)$. Moreover, let $\mathcal{U} \subset \mathcal{M}_{C}$ be the locus of all $(E, D)$ such that the underlying holomorphic vector bundle $E$ is stable. Considering

$$
p: \mathcal{U} \longrightarrow \mathcal{N}
$$

the forgetful morphism that sends any $(E, D)$ to $E$, from the openness of the stability condition (see [Ma, p. 635, Theorem 2.8(B)]) it follows that $\mathcal{U}$ is a Zariski open subset of $\mathcal{M}_{C}$. Note that $p$ is surjective because from [NS] one has that any $V \in \mathcal{N}$ admits a unique logarithmic connection $D_{V}$ singular at $x_{0}$ such that

(a) the residue of $D_{V}$ at $x_{0}$ is $-\frac{\delta}{r} \operatorname{Id}_{V_{x_{0}}}$, and

(b) the monodromy of $D_{V}$ lies in $\mathrm{SU}(r)$.

Moreover, a pair $\left(V, D_{V}\right)$ as above lies in $\mathcal{U}$. In fact, if $D^{\prime}$ is a logarithmic connection on $V$ singular at $x_{0}$ such that $\operatorname{Res}\left(D^{\prime}, x_{0}\right)=-\frac{\delta}{r} \operatorname{Id}_{V_{x_{0}}}$, and the logarithmic connection on $\bigwedge^{r} V=\mathcal{O}_{X}\left(\delta x_{0}\right)$ induced by $D^{\prime}$ coincides with $D_{0}$, then clearly $\left(V, D^{\prime}\right) \in \mathcal{U}$. The space of all logarithmic connections $D^{\prime}$ on $V$ satisfying the conditions (a) and (b) is an affine space for the vector space $H^{0}\left(X, \operatorname{ad}(V) \otimes K_{X}\right)$, where $\operatorname{ad}(V) \subset \operatorname{End}(V)$ is the subbundle of co-rank one defined by the sheaf of endomorphisms of trace zero. Furthermore, $H^{0}\left(X, \operatorname{ad}(V) \otimes K_{X}\right)$ is the fiber of the cotangent bundle $\Omega_{\mathcal{N}}^{1}$ over the point $V \in \mathcal{N}$. Therefore, the morphism $p$ in (2.10) makes $\mathcal{U}$ a torsor over $\mathcal{N}$ for the vector bundle $\Omega_{\mathcal{N}}^{1}$.

From [BM1, p. 301, Lemma 3.1] and [BM2, Lemma 3.1] the complement $\mathcal{M}_{C} \backslash \mathcal{U} \subset \mathcal{M}_{C}$ is of codimension at least two and thus the inclusion map $\iota: \mathcal{U} \hookrightarrow \mathcal{M}_{C}$ produces an isomorphism of Brauer groups

$$
\iota^{*}: \operatorname{Br}\left(\mathcal{M}_{C}\right) \stackrel{\sim}{\longrightarrow} \operatorname{Br}(\mathcal{U}) ;
$$

this follows from "Cohomological purity" [Mi, p. 241, Theorem VI.5.1] (it also follows from [Gr2, p. 292-293]). Since $p$ in (2.10) is a torsor on $\mathcal{U}$ for a vector bundle, and $\mathcal{U}$ is 
simply connected [BBGN, p. 266, Proposition 1.2(b)], from Lemma 2.3 it follows that the map $p$ induces an isomorphism

$$
p^{*}: \operatorname{Br}(\mathcal{N}) \stackrel{\sim}{\longrightarrow} \operatorname{Br}(\mathcal{U}) .
$$

Combining this with (2.11) we get an isomorphism

$$
\left(\iota^{*}\right)^{-1} \circ p^{*}: \operatorname{Br}(\mathcal{N}) \stackrel{\sim}{\longrightarrow} \operatorname{Br}\left(\mathcal{M}_{C}\right) .
$$

We know that $\operatorname{Br}(\mathcal{N})$ is cyclic of order $\tau=$ g.c.d. $(r, \delta)$ [BBGN, p. 267, Theorem 1.8]. Therefore, from (2.12) we conclude that $\operatorname{Br}\left(\mathcal{M}_{C}\right)$ is isomorphic to $\mathbb{Z} / \tau \mathbb{Z}$.

Finally, in order to find a generator of $\operatorname{Br}\left(\mathcal{M}_{C}\right)$, let $\widehat{\mathbb{P}} \longrightarrow X \times \mathcal{N}$ be the universal projective bundle and

$$
\mathbb{P}^{\prime}:=\left.\widehat{\mathbb{P}}\right|_{\{x\} \times \mathcal{N}} \longrightarrow \mathcal{N}
$$

be the projective bundle obtained by restricting $\widehat{\mathbb{P}}$, where $x$ is the point of $X$ in $(2.5)$. The Brauer group $\operatorname{Br}(\mathcal{N})$ is generated by the class of $\mathbb{P}^{\prime}$ [BBGN, p. 267, Theorem 1.8]. The pulled back projective bundle $\left(\operatorname{Id}_{X} \times p\right)^{*} \widehat{\mathbb{P}}$ is identified with the restriction $\left.\widetilde{\mathbb{P}}\right|_{X \times \mathcal{U}}$, where $\widetilde{\mathbb{P}}$ is the projective bundle in (2.4). This implies that $p^{*} \mathbb{P}^{\prime}$ is identified with the restriction $\left.\mathbb{P}\right|_{\mathcal{U}}$. Since the class of $\mathbb{P}^{\prime}$ generates $\operatorname{Br}(\mathcal{N})$, from the isomorphism $\left(\iota^{*}\right)^{-1} \circ p^{*}$ in $(2.12)$ it follows that the class of $\mathbb{P}$ generates $\operatorname{Br}\left(\mathcal{M}_{C}\right)$.

2.2. Analytic Brauer group of representation spaces. Consider now the free group $\Gamma$ generated by $2 g$ elements $\left\{a_{i}, b_{i}\right\}_{i=1}^{g}$, and let

$$
\gamma:=\prod_{i=1}^{g}\left[a_{i}, b_{i}\right]=\prod_{i=1}^{g}\left(a_{i} b_{i} a_{i}^{-1} b_{i}^{-1}\right) \in \Gamma
$$

be the product of the commutators. Then, one may consider the space of all homomorphisms $\operatorname{Hom}(\Gamma, \mathrm{SL}(r, \mathbb{C}))$ from the group $\Gamma$ to $\operatorname{SL}(r, \mathbb{C})$. Let

$$
\operatorname{Hom}^{\delta}(\Gamma, \operatorname{SL}(r, \mathbb{C})) \subset \operatorname{Hom}(\Gamma, \operatorname{SL}(r, \mathbb{C}))
$$

be all such homomorphisms $\rho$ satisfying the condition that

$$
\rho(\gamma)=\exp (2 \pi \sqrt{-1} \delta / r) \cdot I_{r \times r},
$$

where $I_{r \times r}$ is the $r \times r$ identity matrix. The conjugation action of $\mathrm{SL}(r, \mathbb{C})$ on itself produces an action of $\mathrm{SL}(r, \mathbb{C})$ on the variety $\operatorname{Hom}(\Gamma, \mathrm{SL}(r, \mathbb{C}))$. Moreover, this action of $\operatorname{SL}(r, \mathbb{C})$ on $\operatorname{Hom}(\Gamma, \mathrm{SL}(r, \mathbb{C}))$ preserves the above subvariety $\operatorname{Hom}^{\delta}(\Gamma, \mathrm{SL}(r, \mathbb{C}))$. We shall denote by $\overline{\mathcal{R}}$ the geometric invariant theoretic quotient

$$
\overline{\mathcal{R}}:=\operatorname{Hom}^{\delta}(\Gamma, \operatorname{SL}(r, \mathbb{C})) / / \operatorname{SL}(r, \mathbb{C}),
$$

which parametrizes all the closed orbits of $\operatorname{SL}(r, \mathbb{C})$ in $\operatorname{Hom}^{\delta}(\Gamma, \operatorname{SL}(r, \mathbb{C}))$.

The moduli space $\overline{\mathcal{M}}_{C}$ defined in Section 2.1 is biholomorphic to $\overline{\mathcal{R}}$. After fixing a presentation of $\pi_{1}\left(X \backslash\left\{x_{0}\right\}, x\right)$, we have a map $\overline{\mathcal{M}}_{C} \longrightarrow \overline{\mathcal{R}}$ that sends a flat connection to its monodromy representation, and which is a biholomorphism. Indeed, it is the inverse of the map that associates a flat bundle on $X \backslash\left\{x_{0}\right\}$ of rank $r$ to a representation of $\pi_{1}\left(X \backslash\left\{x_{0}\right\}, x\right)$. Note that although both $\overline{\mathcal{M}}_{C}$ and $\overline{\mathcal{R}}$ have natural algebraic structures, the above biholomorphism between them is not an algebraic map. 
A homomorphism $\rho: \Gamma \longrightarrow \mathrm{SL}(r, \mathbb{C})$ is called reducible if $\rho(\Gamma)$ is contained in some proper parabolic subgroup of $\operatorname{SL}(r, \mathbb{C})$, equivalently, if $\rho(\Gamma)$ preserves some proper nonzero subspace of $\mathbb{C}^{r}$ under the standard action of $\operatorname{SL}(r, \mathbb{C})$. If $\rho$ is not reducible, then it is called irreducible.

We shall denote by

$$
\mathcal{R} \subset \overline{\mathcal{R}}
$$

the locus of irreducible homomorphisms in $\overline{\mathcal{R}}$. It is easy to see that $\mathcal{R}$ is contained in the smooth locus of $\overline{\mathcal{R}}$ (in fact, $\mathcal{R}$ coincides with the smooth locus of $\overline{\mathcal{R}}$ ). From the definitions of $\mathcal{M}_{C}$ and $\mathcal{R}$ it follows immediately that the above biholomorphism $\overline{\mathcal{M}}_{C} \stackrel{\sim}{\longrightarrow} \overline{\mathcal{R}}$ produces a biholomorphism

$$
\mathcal{M}_{C} \stackrel{\sim}{\longrightarrow} \mathcal{R}
$$

Let $\mathcal{H} \subset \operatorname{Hom}^{\delta}(\Gamma, \mathrm{SL}(r, \mathbb{C}))$ be the inverse image of $\mathcal{R}$; in other words, $\mathcal{H}$ is the locus of all elements of $\operatorname{Hom}^{\delta}(\Gamma, \operatorname{SL}(r, \mathbb{C}))$ that are irreducible homomorphisms. The quotient map

$$
\mathcal{H} \longrightarrow \mathcal{H} / / \mathrm{SL}(r, \mathbb{C})=\mathcal{R}
$$

makes $\mathcal{H}$ an algebraic principal $\operatorname{PSL}(r, \mathbb{C})$-bundle over $\mathcal{R}$. We shall denote by

$$
\mathbb{P}_{R}:=\mathcal{H} \times{ }^{\mathrm{PSL}(r, \mathbb{C})} \mathbb{C P}^{r-1} \longrightarrow \mathcal{R}
$$

the fiber bundle associated to the principal $\operatorname{PSL}(r, \mathbb{C})$-bundle $\mathcal{H} \longrightarrow \mathcal{R}$ for the standard action of $\operatorname{PSL}(r, \mathbb{C})$ on $\mathbb{C} P^{r-1}$.

The analytic Brauer group of $\mathcal{R}$ is defined to be the equivalence classes of holomorphic principal PGL-bundles on $\mathcal{R}$ where two principal PGL-bundles $P$ and $Q$ are equivalent if there are holomorphic vector bundles $V$ and $W$ on $\mathcal{R}$ such that the two principal PGLbundles $P \otimes \mathbb{P}(V)$ and $Q \otimes \mathbb{P}(W)$ are isomorphic. Moreover, the analytic cohomological Brauer group of $\mathcal{R}$ is the torsion part of $H^{2}\left(\mathcal{R}, \mathcal{O}_{\mathcal{R}}^{*}\right.$ ) (see [Sc]). Let $\operatorname{Br}_{\text {an }}(\mathcal{R})$ (respectively, $\mathrm{Br}_{\text {an }}^{\prime}(\mathcal{R})$ ) denote the analytic Brauer group (respectively, analytic cohomological Brauer group) of $\mathcal{R}$. Then, from [Sc, p. 878] one has that

$$
\mathrm{Br}_{\text {an }}(\mathcal{R}) \subset \operatorname{Br}_{\text {an }}^{\prime}(\mathcal{R})
$$

Theorem 2.5. The analytic cohomological Brauer group $\mathrm{Br}_{\mathrm{an}}^{\prime}(\mathcal{R})$ is isomorphic to a quotient of the cyclic group $\mathbb{Z} / \tau \mathbb{Z}$, where $\tau=$ g.c.d. $(r, \delta)$. Moreover, the group $\operatorname{Br}_{\text {an }}(\mathcal{R})$ is generated by the class of the projective bundle $\mathbb{P}_{R}$ in (2.15).

Proof. From the biholomorphism in $(2.14)$, the group $\operatorname{Br}_{\text {an }}^{\prime}(\mathcal{R})$ coincides with the analytic Brauer group $\operatorname{Br}_{\text {an }}^{\prime}\left(\mathcal{M}_{C}\right)$ of the stable moduli space $\mathcal{M}_{C}$. Moreover, the forgetful map $\operatorname{Br}^{\prime}\left(\mathcal{M}_{C}\right) \longrightarrow \operatorname{Br}_{\text {an }}^{\prime}\left(\mathcal{M}_{C}\right)$ is surjective [Sc, p. 879, Proposition 1.3]. Then, since $\operatorname{Br}^{\prime}\left(\mathcal{M}_{C}\right)=\operatorname{Br}\left(\mathcal{M}_{C}\right)$, we conclude that $\operatorname{Br}_{\text {an }}^{\prime}(\mathcal{R})$ is a quotient of $\operatorname{Br}^{\prime}\left(\mathcal{M}_{C}\right)$. Therefore, from the first part of Theorem 2.4 it follows that $\operatorname{Br}_{\text {an }}^{\prime}(\mathcal{R})$ is a quotient of the cyclic group $\mathbb{Z} / \tau \mathbb{Z}$.

The isomorphism in (2.14) takes the projective bundle $\mathbb{P}_{R}$ constructed in (2.15) holomorphically to the projective bundle $\mathbb{P}$ of $(2.5)$. Therefore, from the second part of Theorem 2.4 it follows that $\operatorname{Br}_{\text {an }}^{\prime}(\mathcal{R})$ is generated by the class of $\mathbb{P}_{R}$. 
Note that whilst the natural homomorphism $\operatorname{Br}_{\text {an }}(\mathcal{R}) \longrightarrow \operatorname{Br}_{\text {an }}^{\prime}(\mathcal{R})$ is injective [Sc, p. 878], Theorem 2.5 implies that this homomorphism is surjective and thus the following corollary is proved.

Corollary 2.6. The analytic Brauer group $\mathrm{Br}_{\text {an }}(\mathcal{R})$ coincides with $\mathrm{Br}_{\text {an }}^{\prime}(\mathcal{R})$.

Regarding the above Corollary 2.6 it should be clarified that the analog of Gabber's theorem, which would say that the Brauer group coincides with the cohomological Brauer group, is not available in the analytic category.

2.3. Brauer group of moduli spaces of Higgs bundles. We shall now consider Higgs bundles on a compact Riemann surface. As in Section 2.1, consider a compact connected Riemann surface $X$ of genus $g \geq 3$, and $x_{0} \in X$ a base point. Let $\mathcal{M}_{H}$ denote the moduli space of stable Higgs bundles on $X$ of the form $(E, \Phi)$, where $E$ is a holomorphic vector bundle of rank $r$ with $\bigwedge^{r} E=\mathcal{O}_{X}\left(\delta x_{0}\right)$, and $\Phi$ is a Higgs field on $X$ with $\operatorname{Tr}(\Phi)=0$. The moduli space $\mathcal{M}_{H}$ is an irreducible smooth complex quasiprojective variety of dimension $2\left(r^{2}-1\right)(g-1)$.

Consider the moduli space $\mathcal{N}$ from (2.10), for which the total space of the algebraic cotangent bundle $T^{*} \mathcal{N}$ is embedded in in $\mathcal{M}_{H}$ as a Zariski open subset. The codimension of the complement $\mathcal{M}_{H} \backslash T^{*} \mathcal{N}$ is at-least two [Hi]. Therefore, by purity of cohomology, and Lemma 2.3, one has that

$$
\operatorname{Br}(\mathcal{N})=\operatorname{Br}\left(T^{*} \mathcal{N}\right)=\operatorname{Br}\left(\mathcal{M}_{H}\right)
$$

as before we use that $\mathcal{N}$ is simply connected.

Hence we have the following:

Proposition 2.7. The Brauer group $\operatorname{Br}\left(\mathcal{M}_{H}\right)$ is the cyclic group of order g.c.d. $(r, \delta)$. Furthermore, $\operatorname{Br}\left(\mathcal{M}_{H}\right)$ is generated by the class of the projective bundle on $\mathcal{M}_{H}$ obtained by restricting to $\{x\} \times \mathcal{M}_{H}$ the universal projective bundle on $X \times \mathcal{M}_{H}$.

\section{Generalizations to principal Bundles}

Let $G$ be a semisimple simply connected affine algebraic group defined over $\mathbb{C}$. The topological types of principal $G$-bundles on $X$ are parametrized by $\pi_{1}(G)$, which is a finite abelian group. Let $\overline{\mathcal{M}}_{C}(G)$ denote the moduli space of pairs of the form $\left(E_{G}, D\right)$, where $E_{G}$ is a topologically trivial holomorphic principal $G$-bundle on $X$, and $D$ is a holomorphic connection on $E_{G}$. Following the notation from the previous sections, let

$$
\mathcal{M}_{C}(G) \subset \overline{\mathcal{M}}_{C}(G)
$$

be the smooth locus of $\overline{\mathcal{M}}_{C}(G)$.

The center of $G$ will be denoted by $Z(G)$. A stable principal $G$-bundle is called regularly stable if its automorphism group coincides with $Z(G)$. We shall denote by $\mathcal{N}_{G}$ the moduli space of regularly stable principal $G$-bundles on $X$ that are topologically trivial. Recall from $[\mathrm{BHol}]$ that the Brauer group $\operatorname{Br}\left(\mathcal{N}_{G}\right)$ can be identified with the dual group 
$Z(G)^{\vee}=\operatorname{Hom}\left(Z(G), \mathbb{C}^{*}\right)$, and $\operatorname{Br}\left(\mathcal{N}_{G}\right)$ is generated by the tautological $Z(G)$-gerbe on $\mathcal{N}_{G}$ defined by the moduli stack of regularly stable topologically trivial principal $G^{-}$ bundles on $X$. Note that given any homomorphism $Z(G)^{\vee} \longrightarrow \mathbb{G}_{m}$, using extension of structure group the above $Z(G)$-gerbe produces a $\mathbb{G}_{m}$-gerbe on $\mathcal{N}_{G}$.

Proposition 3.1. The Brauer group $\operatorname{Br}\left(\mathcal{M}_{C}(G)\right)$ is isomorphic to the dual group $Z(G)^{\vee}$ and is generated by the tautological $Z(G)$-gerbe on $\mathcal{M}_{C}(G)$.

Proof. A straight-forward generalization of the proof of Theorem 2.4 proves the proposition. We note that $\mathcal{N}_{G}$ is simply connected [BLR, p. 416, Theorem 1.1], hence Lemma 2.3 is applicable.

Similarly, the (analytic) Brauer group computations in Theorem 2.5 and Section 2.3 extend to $G$.

\section{LANGLANDS DUALITY AND BRAUER GROUPS}

As previously, suppose that $G$ is simply connected and let ${ }^{L} G$ denote the Langlands dual group. There is a canonical isomorphism $\pi_{1}\left({ }^{L} G\right) \cong Z(G)^{\vee}$, which can be seen from the root-theoretic construction of the Langlands dual. We shall denote by $\mathcal{M}_{H}(G)$ and $\mathcal{M}_{H}\left({ }^{L} G\right)$ the moduli spaces of Higgs bundles for the groups $G$ and ${ }^{L} G$ respectively.

It is known that the connected components of $\mathcal{M}_{H}\left({ }^{L} G\right)$ correspond to $\pi_{1}\left({ }^{L} G\right)$, by taking the topological class of the underlying principal bundle. Recall that the moduli spaces $\mathcal{M}_{H}(G)$ and $\mathcal{M}_{H}\left({ }^{L} G\right)$ satisfy SYZ mirror symmetry, that is, they are dual special Lagrangian torus fibrations over a common base [DoPa]. Under this duality, the choice of a connected component of $\mathcal{M}_{H}\left({ }^{L} G\right)$ corresponds to fixing a $\mathbb{C}^{*}$-gerbe on $\mathcal{M}_{H}(G)$. Namely, given a class in $\pi_{1}\left({ }^{L} G\right) \cong Z(G)^{\vee}$, we obtain from the universal $G / Z(G)$-bundle on $\mathcal{M}_{H}(G)$ the corresponding $\mathbb{C}^{*}$-gerbe. Our computations show that every class in $\operatorname{Br}\left(\mathcal{M}_{H}(G)\right)$ is accounted for by this correspondence.

\section{ACKNOWLEDGEMENTS}

We are very grateful to T. J. Ford for a helpful correspondence. We thank the Institute for Mathematical Sciences in the National University of Singapore for hospitality while this work was being completed. IB acknowledges support of a J. C. Bose Fellowship. This research was completed with support from U.S. National Science Foundation grants DMS 1107452, 1107263, 1107367 sRNMS: GEometric structures And Representation varieties (the GEAR Network). The work of LPS is partially supported by NSF DMS-1509693.

\section{REFERENCES}

[BBGN] V. Balaji, I. Biswas, O. Gabber and D. S. Nagaraj, Brauer obstruction for a universal vector bundle, Comp. Ren. Math. Acad. Sci. Paris 345 (2007), 265-268.

[BM1] I. Biswas and V. Muñoz, The Torelli theorem for the moduli spaces of connections on a Riemann surface, Topology 46 (2007), 295-317. 
[BM2] I. Biswas and V. Muñoz, Torelli theorem for moduli spaces of SL $(r, \mathbb{C})$-connections on a compact Riemann surface, Commun. Contemp. Math. 11 (2009), 1-26.

[BHof] I. Biswas and N. Hoffmann, A Torelli theorem for moduli spaces of principal bundles over a curve, Ann. Inst. Fourier 62 (2012), 87-106.

[BHol] I. Biswas and Y. I. Holla, Brauer group of moduli of principal bundles over a curve, Jour. Reine Angew. Math. 677 (2013), 225-249.

[BLR] I. Biswas, S. Lawton and D. Ramras, Fundamental groups of character varieties: surfaces and tori, Math. Zeit. 281 (2015), 415-425.

$[\mathrm{dJ}] \quad$ A.J. de Jong, A result of Gabber, http://www.math.columbia.edu/ dejong/papers/2-gabber.pdf.

[De] P. Deligne, Equations différentielles à points singuliers réguliers, Lecture Notes in Mathematics, Vol. 163, Springer-Verlag, Berlin-New York, 1970.

[DoPa] R. Donagi and T. Pantev, Langlands duality for Hitchin systems, Invent. Math. 189 (2012), 653-735.

[Fo] T. J. Ford, On the Brauer group of a localization, Jour. Alg. 147 (1992), 365-378.

[Ga] O. Gabber, Some theorems on Azumaya algebras, in The Brauer group (Sem., Les Plans-surBex, 1980), pp. 129-209, Lecture Notes in Math., 844, Springer, Berlin-New York, 1981.

[Gr1] A. Grothendieck, Le groupe de Brauer, I, in Dix exposés sur la cohomologie des schémas, pp. 46-66, Advanced Studies in Pure Mathematics, 3, North-Holland, Amsterdam, 1968.

[Gr2] A. Grothendieck, Le groupe de Brauer, I, in Dix exposés sur la cohomologie des schémas, pp. 67-87, Advanced Studies in Pure Mathematics, 3, North-Holland, Amsterdam, 1968.

[Gr3] A. Grothendieck, Le groupe de Brauer, I, in Dix exposés sur la cohomologie des schémas, pp. 88-188, Advanced Studies in Pure Mathematics, 3, North-Holland, Amsterdam, 1968.

[Ha] A. Hatcher, Algebraic topology, Cambridge University Press, Cambridge, 2002.

[Hi] N. J. Hitchin, Stable bundles and integrable systems, Duke Math. Jour. 54 (1987), 91-114.

[Ho] R. T. Hoobler, When is $\operatorname{Br}(X)=\operatorname{Br}^{\prime}(X)$ ? in Brauer groups in ring theory and algebraic geometry (Wilrijk, 1981), pp. 231-244, Lecture Notes in Math., 917, Springer, Berlin-New York, 1982.

[Ma] M. Maruyama, Openness of a family of torsion free sheaves, Jour. Math. Kyoto Univ. 16 (1976), 627-637.

[Mi] J. S. Milne, Étale cohomology, Princeton Mathematical Series, 33, Princeton University Press, 1980.

[NS] M. S. Narasimhan and C. S. Seshadri, Stable and unitary vector bundles on a compact Riemann surface, Ann. of Math. 82 (1965), 540-567.

[Oh] M. Ohtsuki, A residue formula for Chern classes associated with logarithmic connections, Tokyo Jour. Math. 5 (1982), 13-21.

[Sc] S. Schröer, Topological methods for complex-analytic Brauer groups, Topology 44 (2005), 875894.

School of Mathematical Sciences, The University of Adelaide, Adelaide SA 5005, AusTRALIA

E-mail address: david.baraglia@adelaide.edu.au

School of Mathematics, Tata Institute of Fundamental Research, Homi Bhabha Road, BOMBAY 400005, INDIA

E-mail address: indranil@math.tifr.res.in

Department of Mathematics, University of Illinois, Chicago, IL 60607, USA

E-mail address: schapos@illinois.edu 